Muséologies

Les cahiers d'études supérieures

muséologies

\title{
Comment donner envie d'apprendre au musée ? Un exemple : le Service éducatif des musées des arts décoratifs
}

\section{Adèle Robert}

Volume 3, numéro 2, printemps 2009

URI : https://id.erudit.org/iderudit/1033575ar

DOI : https://doi.org/10.7202/1033575ar

Aller au sommaire du numéro

Éditeur(s)

Association Québécoise de Promotion des Recherches Étudiantes en

Muséologie (AQPREM)

ISSN

1718-5181 (imprimé)

1929-7815 (numérique)

Découvrir la revue

Citer cet article

Robert, A. (2009). Comment donner envie d'apprendre au musée ? Un exemple : le Service éducatif des musées des arts décoratifs. Muséologies, 3(2), 214-223.

https://doi.org/10.7202/1033575ar d'utilisation que vous pouvez consulter en ligne. 
Article seize

\section{Comment donner envie d'apprendre au musée?}

\section{Un exemple : Le Service éducatif des musées}

\section{des arts décoratifs}

DIPLOMMÉE ĖS BEAUX-ARTS DE L'UNIVERSITÉ DE CALIFORNIE À BERKELEY ET EN COMMUNICATION VISUELLE DE LUNIVERSITÉ DE CINCINNATI (OHIO), ADËLE ROBERT A PRIS PART À LATELIER DES ENFANTS AU CENTRE GEORGES POMPIDOU (PARIS); AÉTÉ CHEF DE PROJET POUR LA CONCEPTION ET LA REALISATION DE LINVENTORIUM ("LA CITÉ DES ENFANTS") ET DE LA CITÉ DES SCIENCESET DE LINDUSTRIE DE LA VILLETIE (PARIS); ELLE A COLLABORÉ À DE NOMBREUX PROJETS EN TANT QUE CONSUITANT CONCEPTEUR, NOTAMMENT LE MUSÉE DE STELLAMATUTINA (ULE DE LA RÉUNION), LEMUSEEE DES MONUMENTS FRANÇAIS, LA GRANDE BIBLIOTHÈQUE DE FRANCE, LES ARCHIVES DU MONDE DU TRAVALL (ROUBAIX); ELIE AÉTÉ CHEF DU SERVICE ÉDUCATIF DES QUATRE MUSÉES DES ARTS DÉCORATIFS (PARIS) ET MEMBRE DU BUREAU EXÉCUTIF DU COMITÉ D'ÉDUCATION ET DACTION CULTURELLE DU CONSEIL INTERNATIONAL DES MUSÉES (ICOM). [robertadele@free.fr] 


\section{Les arts décoratifs Présentation}

Organisme privé, association loi 1901 reconnue d'utilité publique, les Arts Décoratifs ont été créés en 1882 dans le sillage des expositions universelles par des collectionneurs soucieux de valoriser les beaux-arts appliqués et de tisser des liens entre industrie et culture, création et production.

Institution originale et plurielle, les Arts Décoratifs poursuivent les objectifs qui ont présidé à leur fondation, «entretenir en France la culture des arts qui poursuivent la réalisation du beau dans l'utile", et maintiennent des liens étroits avec le monde industriel, établissant de nombreux partenariats avec des entreprises qui exercent leurs activités dans des domaines variés.

Longtemps connus sous la dénomination «Union centrale des arts décoratifs (Ucad)", les Arts Décoratifs ont modernisé leur image en décembre 2004 en restant fidèles à leur vocation d'origine : exercer des missions de conservation des collections et de diffusion culturelle, d'éducation artistique et de formation de professionnels, de soutien à la création.

Avec ses quatre musées - le musée des Arts décoratifs, le musée de la Mode et du Textile, le musée de la Publicité, le musée Nissim de Camondo-, la bibliothèque des Arts décoratifs est un pôle d'enseignement; les Arts Décoratifs disposent d'un ensemble de collections permanentes d'une extrême richesse et d'une grande valeur éducative.

Les collections présentent tous les aspects de la production artistique, dans tous les domaines des arts décoratifs, et illustrent les techniques les plus diverses : arts du bois (sculpture, mobilier, boiseries), du métal (orfevrerie, 
fer, bronze, étain), de la céramique, du verre, du cuir (écrins, reliures), de la peinture, mais aussi celles, plus modestes, des marqueteries de paille, des broderies de perles, des tôles peintes...

Étroitement liées au savoir-faire des artisans, aux matériaux et aux techniques, les oeuvres de l'ensemble des Arts Décoratifs sont particulièrement adaptées à une démarche pédagogique active qui font appel à la participation des jeunes par la manipulation, le toucher, l'écoute, le montage ou le démontage des éléments techniques, les jeux d'assemblage, etc.

\section{Un service éducatif au sein d'un service des publics d'une institution culturelle...}

Les Arts Décoratifs sont parmi les premiers en France à avoir créé un service du musée dédié au public. Dès 1951, des visites guidées et des conférences sont conçues pour les enfants et les adultes afin de rendre les collections permanentes et les expositions temporaires accessibles au plus grand nombre.

Par une politique dynamique, le Service éducatif s'efforce de répondre à la mission qui lui a été confiée : développer un intérêt pour les objets des musées et contribuer à la formation du visiteur de demain.

La mise en oeuvre de nombreuses propositions pédagogiques originales au cours des années a permis d'élargir de manière significative son champ d'action et son audience. La démarche et la réflexion pédagogique que nous avons développées en relation avec des chefs-d'oeuvre des arts décoratifs sont le fruit d'une longue réflexion et le résultat des recherches et de l'expérience de la conception et de la réalisation des musées pour les enfants et des actions pédagogiques.

Pour développer ce service, nous avons clairement exprimé dès 1990 les objectifs pédagogiques, inventé de nouveaux projets pédagogiques (notamment des visites ateliers) et, très progressivement, programmé des activités pour les jeunes de quatre à 12 ans, d'abord, pendant certaines vacances scolaires et ensuite pour les groupes scolaires jusqu'à 18 ans.

Dans le but de préciser notre approche, nous avons aussi analysé les objectifs de l'éducation formelle (l'école) et ceux de l'éducation non formelle (autres activités) et nous avons tenté de comprendre en quoi un service éducatif de musée est différent de l'école. 


\section{Des objectifs pédagogiques}

Compte tenu des différents niveaux d'intérêt et des stades d'initiation de chacun, le Service éducatif a pour objectifs :

- de permettre aux jeunes visiteurs d'explorer le musée de façon autonome ou en groupe,

- de les aider à s'interroger sur ce qu'ils voient, ce qu'ils ressentent, afin de susciter des curiosités nouvelles,

- de les amener à mieux comprendre les relations entre les contextes artistiques et historiques,

- de prolonger un cours ou de lancer des travaux interdisciplinaires intégrant la culture artistique dans le programme scolaire.

\section{"J'entends et j’oublie, je vois et je me souviens, je fais et je comprends. "}

Ce proverbe de Confucius exprime l'esprit de la démarche pédagogique du Service éducatif.

\section{La démarche pédagogique}

Tout projet prend ses racines dans les collections permanentes ou les expositions temporaires des musées des arts décoratifs.

De manière pratique, la démarche pédagogique s'organise en projets pédagogiques thématiques qui s'inscrivent dans un module pédagogique qui les prolonge et les complète.

La thématique est choisie par rapport aux intérêts du jeune auquel elle s'adresse, pour sa capacité à faire appel à la participation active du jeune visiteur (toucher, goûter, sentir, monter ou démonter un objet technique ou une réalisation en atelier). Elle prend en considération les programmes scolaires du niveau concerné.

Lors de la conception d'un projet pédagogique thématique, il est important de rester attentif au processus d'acquisition des connaissances des jeunes.

Parmi ceux qui se sont intéressés aux différents aspects de l'éducation, le psychologue suisse Jean Piaget a mis en évidence la spécificité des processus cognitifs des jeunes 5 enfants dès 1919. Il a montré d'une façon scientifique 
que les enfants raisonnent d'une manière différente de celle des adultes, mais que leurs pensées ont un schéma logique.

Il est donc possible de concevoir des projets pédagogiques pour les plus jeunes dans les musées. Les projets pour les enfants dès quatre ans sont ainsi construits autour d'une approche de l'objet qui est différente de celle de l'adulte et de l'adolescent.

La démarche s'appuie également sur les connaissances en sciences de l'éducation : les méthodes pédagogiques de Maria Montessori et de Célestin Freinet, les connaissances en histoire de l'art, le design, les techniques et une longue pratique pédagogique avec les jeunes.

Les projets pédagogiques destinés aux enfants ne doivent pas faire l'objet d'explications longues qui mettent en jeu des phénomènes et du vocabulaire, ni de rapports complexes qui sont mieux adaptés à un public d'adultes.

Plutôt que d'étaler une suite d'idées théoriques ou d'expliquer des oeuvres en essayant de les faire comprendre, nous avons essayé de créer des situations dans lesquelles les enfants ont l'occasion d'expérimenter. Ainsi, chaque fois que c'était possible, nous avons développé (inventé) un matériel pédagogique permettant à l'enfant d'explorer librement un sujet par l'expérimentation (ex : les boîtes à Lumière pour la visite atelier «Les lumières de la Renaissance " ou les petits carnets interactifs pour l'exposition temporaire du Département des jouets, Histoires d'ours, et un travail en atelier sur les expressions des visages des jouets qui permet à l'enfant de découvrir que "chacun est différent»).

Comme la majorité des activités et des présentations sont originales, le développement d'une activité pour l'enfant ou l'adolescent passe par plusieurs étapes. Une importante partie de ce travail est l'observation de l'enfant pendant ou après l'utilisation d'un matériel pédagogique, cela étant considéré comme un "prototype ". Cette " évaluation " permet de modifier si nécessaire ou d'adapter le projet avant de le proposer de façon permanente aux visiteurs. Cette démarche expérimentale a permis de créer un esprit d'équipe. Au cours des années et d'un travail de recherche pédagogique, plusieurs situations éducatives ont pu être identifiées :

Des situations de conflit - qui concernent la relation entre des connaissances de l'enfant et des nouvelles informations qui vont modifier sa compréhension. Exemple : visite atelier pratique « Les couleurs au Moyen Âge " pour les quatre à dix ans. L'enfant fait de la peinture avec de la poudre et de l'eau. Ca ne marche pas. Avec l'aide du jaune d'oeuf, ça tient. Ensuite, l'enfant reçoit des explications sur la peinture à tempera. 
Des situations d'essais et erreurs - qui provoquent un déséquilibre entre ce que l'enfant croit savoir et le phénomène réel qui va modifier la compréhension. Exemple : Visite atelier pratique "Une façon d'imprimer » pour les quatre à six ans. L'enfant imprime un motif décoratif et découvre la technique de la séparation des couleurs en imprimerie du XIX` siècle.

Des situations de problème - qui demandent des expérimentations conduisant à une solution. Exemple : Visite atelier pratique "Le Bauhaus", pour les neuf à 18 ans. En relation avec le mobilier de Mies Van de Rohe, on demande aux jeunes de "faire plus avec moins ", en réalisant la maquette d'une chaise qui utilise le moins de matériel possible pour porter un poids.

Selon notre expérience, une activité au musée pour les enfants ou pour les adolescents doit :

- intéresser l'enfant ou l'adolescent;

- maintenir son intérêt assez longtemps pour l'inciter à réaliser une ou plusieurs des opérations suivantes :

1] interaction sensorielle ou motrice

(observer, toucher, écouter, etc.),

2] interaction systématique expérimentale (réalisation d'une expérimentation systématique avec analyse des résultats... situations d'exploration, situations amenant des modifications de l'image mentale préexistante, situations problèmes/solutions);

- communiquer dans un domaine spécifique des arts décoratifs (mobilier, mode, graphisme...), de la technique ou de l'industrie ;

- transmettre des concepts artistiques, historiques ou techniques, une compétence technique ou du vocabulaire;

- stimuler l'intérêt des enfants pour les inciter à voir d'autres présentations sur le même thème.

Ces critères importants ont permis de sélectionner des thèmes pour les diverses activités dédiées au jeune public de quatre à 18 ans dans les musées des arts décoratifs. De nouveaux projets pédagogiques ont été créés systématiquement par le Service éducatif pour les collections permanentes des musées et pour les expositions temporaires : des visites guidées ont été proposées systématiquement pour les nouvelles expositions. 
Des méthodes de travail

\section{La fiche pédagogique...}

Chaque projet pédagogique et thématique fait l'objet d'une étude détaillée et d'un document écrit (une fiche pédagogique). Ces fiches permettent aux conférenciers et aux animateurs de s'accorder sur une même démarche conceptuelle, de réaliser le même parcours dans le musée, d'évaluer chaque projet et éventuellement d'y apporter des modifications après une expérimentation avec les jeunes.

\section{...qui intègre l'expérimentation et l'évaluation}

Par ailleurs, ces projets pédagogiques « expérimentaux» doivent être considérés comme des projets de recherche en psychologie de l'enfant et ou de l'adolescent, notamment sur l'acquisition des connaissances dans un lieu d'éducation « informelle» comme le musée (l'école étant un lieu d'éducation "formelle", avec vérification des connaissances, passage d'un niveau à l'autre...). Les liens créés, notamment avec le Département de psychologie de l'Université de Nanterre pour la formation continue des conférenciers, sont à poursuivre.

...et un budget équilibré

La rédaction détaillée des fiches pédagogiques permet également de déterminer le budget détaillé du projet et de le respecter. 


\section{En conclusion}

Un service éducatif de musée doit effectivement rester " ouvert " pour répondre à des objectifs nouveaux pouvant apparaître à travers le musée. Selon notre expérience personnelle, c'est par le biais de l'observation attentive des enfants et des jeunes et l'évaluation continuelle des actions pédagogiques par les groupes et par le grand public que l'on pourrait trouver des moyens pour aller au-delà d'une première démarche. Cela permettrait en permanence d'évaluer et d'adapter cette " éducation par le musée " aux nouvelles demandes, aux récentes connaissances artistiques, techniques et pédagogiques. 


\section{Bibliographie complémentaire}

\section{ART ET DESIGN, TECHNIQUE, SCIENCE}

BRUNHAMMER, YVonne. Le beau dans l'utile, un musée pour les arts décoratifs. Paris : Gallimard, coll. "Découvertes", 1992, n 145.

FRANCASTEL, Pierre. Art et technique. Paris : Denoël, 1983.

LEROI-GOURHAN, Élaine. "Le geste et la parole, technique et langage". Science d'Aujourd'hui. Paris : Albin Michel, 1964.

\section{ÉDUCATION ET PSYCHOLOGIE}

CASANOVA, Laetitia, Serge CHAUMIER et Marie-Claire HABIB. Les accompagnateurs de la Cité des Enfants. Département Évaluation et Prospective, Cité des sciences et de l'industrie, 1995.

DEWEY, John. "The Need of a Theory Experience". Experience and Education. Kappa Delta Pi Lectures Series. New York : Collier Books, 1938, p. 90-94.

HEIN, George. Learning in the Museum. Londres : Routledge, 1998.

HEIN, George. "Comment les enfants se comportent-ils dans les musées?". ICOM Éducation, nos 12-13, ICOM (Conseil international des musées) et CECA (Comité d'éducation et de l'action culturelle), 1991, p. 52-57.

HEIN, George. Collaborative Humanities Project Summary Evaluation Report. Cambridge (MA) : Program Evaluation and Research Group, Lesley College, 1980.

HEIN, George. Evaluation Report, Museum of Science and Sommerville School System Collaborative Pilot Project. Cambridge (MA) : Program Evaluation and Research Group, Lesley College, 1964.

HEUMANN GURIAN, Élaine. Civilizing the Museum: The Collected Writings. New York : Routledge, 2006.

ICOM (Conseil international des musées), et CECA (Comité d'éducation et de l'action culturelle). Conférence "The Museum Exhibition as a Tool in Educational Work". Svendborg/Copenhagen, 1982, p. 17-20.

PIAGET, Jean. Psychologie et pédagogie. Paris : Gallimard, coll. "Folio Essai», no 91, 1991. PIAGET, Jean. La psychologie de l'intelligence. Paris : Armand Colin, 1947.

SCREVEN, Chandlar. The Measurement and Facilitation of Learning in the Museum Environment: An Experimental Analysis. Washington (DC) : Smithsonian Institution Press, 1976.

\section{MUSÉOLOGIE}

RIVIĖRE, Georges-Henri. "La Muséologie ». Cours de Muséologie / Textes et Témoignages : Dunod, Bordas, 1989. 
Throughout my professional experience in the conception, realization, and management of education departments in different types of museums in France, and through cooperating with various teams, I was able to develop a pedagogical approach that enables children from approximately six to eleven years of age, not only to learn by way of active participation, but to enjoy the museum and learning experience. In fact, caution must be exercised in designing programs for children in museums, as they can quickly become simple gadgets that will interest children and even amuse them, but that will not have the expected cognitive impact. I was asked by the École du Louvre to prepare a class for adult students illustrating how this approach was adapted to the Musée des Arts décoratifs in Paris, where I was in charge of education services from 1990 to 2008. This passionate museum, also closely related to craftsmen's know-how, as well as to materials and techniques, is particularly adapted to a pluridisciplinary and active approach to learning, as the collections stimulate handling, touching, listening, as well as assembling and disassembling objects, etc. From my experience, it is notably through training in education sciences and the attentive observation of children and youth, as well as formative evaluation of pedagogical actions with groups and the general public, that we will find a way to go beyond the initial approach and develop projects that will truly correspond to our goals. 